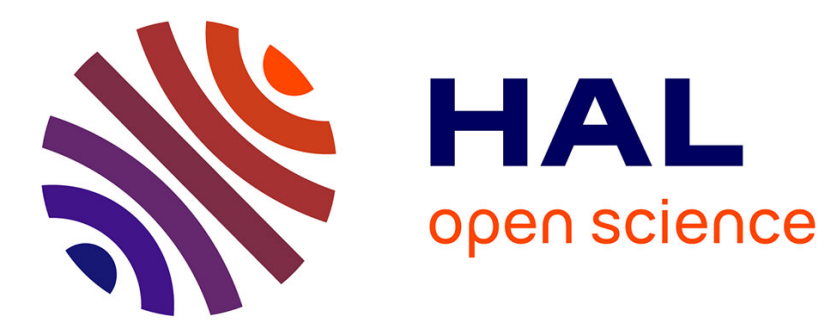

\title{
Synthetic biological circuit tested in spaceflight
}

Rebekah Kitto, Yadvender Dhillon, James Bevington, Mera Horne, Philippe

Giegé, Laurence Drouard, Dimitri Heintz, Claire Villette, Nicolas Corre, Mathilde Arrivé, et al.

\section{- To cite this version:}

Rebekah Kitto, Yadvender Dhillon, James Bevington, Mera Horne, Philippe Giegé, et al.. Synthetic biological circuit tested in spaceflight. Life Sciences in Space Research, 2020, 10.1016/j.lssr.2020.09.002 . hal-03011574

\section{HAL Id: hal-03011574 https://hal.science/hal-03011574}

Submitted on 18 Nov 2020

HAL is a multi-disciplinary open access archive for the deposit and dissemination of scientific research documents, whether they are published or not. The documents may come from teaching and research institutions in France or abroad, or from public or private research centers.
L'archive ouverte pluridisciplinaire HAL, est destinée au dépôt et à la diffusion de documents scientifiques de niveau recherche, publiés ou non, émanant des établissements d'enseignement et de recherche français ou étrangers, des laboratoires publics ou privés. 


\title{
Synthetic biological circuit tested in spaceflight
}

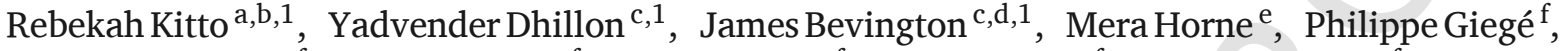 \\ Laurence Drouard $^{\mathrm{f}}$, Dimitri Heintz ${ }^{\mathrm{f}}$, Claire Villette ${ }^{\mathrm{f}}$, Nicolas Corre ${ }^{\mathrm{f}}$, Mathilde Arrivé ${ }^{\mathrm{f}}$, Mike Manefield ${ }^{\mathrm{d}, \mathrm{g}}$, \\ Robert Bowman $^{\mathrm{h}}$, Jean-Jacques Favier ${ }^{\mathrm{c}}$, Barnaby Osborne ${ }^{\mathrm{c}}$, Chris Welch ${ }^{\mathrm{c}, *}$, Christopher P. McKay ${ }^{\mathrm{e}, *}$, \\ Ming Hammond ${ }^{\mathrm{a}, \mathrm{b}, *}$ \\ a Department of Chemistry, and Henry Eyring Center for Cell and Genome Sciences, University of Utah, Salt Lake City, UT, USA \\ ${ }^{\mathrm{b}}$ Department of Chemistry, University of California Berkeley, Berkeley, CA, USA \\ ${ }^{\mathrm{c}}$ International Space University, Strasbourg, France \\ d School of Chemical Engineering, University of New South Wales, Sydney, NSW, Australia \\ e Space Science Division, NASA Ames Research Center, Moffett Field, CA, USA \\ ${ }^{\mathrm{f}}$ Institut de Biologie Moléculaire des Plantes-CNRS, Université de Strasbourg, Strasbourg, France \\ ${ }^{g}$ School of Civil Engineering, University of New South Wales, Sydney, NSW, Australia \\ ${ }^{\mathrm{h}}$ Space Biosciences Division, NASA Ames Research Center, Moffett Field, CA, USA
}

\section{A B S T R A C T}

Synthetic biology has potential spaceflight applications yet few if any studies have attempted to translate earth-based synthetic biology tools into spaceflight. An exogenously inducible biological circuit for protein production in Arabidopsis thaliana, pX7-AtPDSi (Guo et al. 2003), was flown to ISS and functionally investigated. Seedlings were grown in a custom built $1.25 \mathrm{U}$ plant greenhouse. Images recorded during the experiment show that leaves of pX7-AtPDSi seedlings photobleached as designed while wild type Col-0 leaves did not, which reveals that the synthetic circuit led to protein production during spaceflight. Polymerase chain reaction analysis post-flight also confirms that the Cre/LoxP (recombination system) portions of the circuit were functional in spaceflight. The subcomponents of the biological circuit, estrogen-responsive transcription factor XVE, Cre/LoxP DNA recombination system, and RNAi post-transcriptional gene silencing system now have flight heritage and can be incorporated in future designs for space applications. To facilitate future plant studies in space, the full payload design and manufacturing files are made available.

\section{Introduction}

Space exploration presents many challenges such as extreme environments, long lead times for supply chains, and risks arising from unknown unknowns. Future mission plans will likely aim for self-sufficient crews able to implement solutions that can be adaptable to many situations. For example, 3D printers can build diverse components from the same starting materials. Such devices are currently under development for producing tools and even habitats from local materials for future exploration missions (Cesaretti et al., 2014; Wong and Pfahnl, 2014).

Synthetic biology is comparable to a $3 \mathrm{D}$ printer in its ability to produce numerous biomolecules from the same starting materials (Menezes et al., 2015). Of particular interest are medicines that have reduced shelf lives in space (Du et al., 2011) and shelf lives shorter than proposed mission architectures for a human mission to Mars. Additionally, the ability to produce in-situ leads to reduced spacecraft masses because not every medicine or biomolecule that could possibly be required through the mission has to be taken. Plants hold particular promise for bioproduction because they already can play a critical role in life support systems through cycling $\mathrm{CO}_{2}$ and producing food for astronauts (Poulet et al., 2016). The application of synthetic biology to plants could further enhance their value to human exploration missions by making them multi-functional (Llorente et al., 2018). One can envision plants designed to produce vitamins or medicines (Zhang et al., 2017) critical to astronaut health on demand. These would be consumed with a normal plant-based meal. An alternate application includes plants that respond to toxins by indicating their presence and sequestering them or converting them to less hazardous compounds. Using an engineering approach, crops could be enhanced for these on-demand applications.

\footnotetext{
* Corresponding authors.

E-mail addresses: chris.welch@isunet.edu (C. Welch); chris.mckay@nasa.gov (C.P. McKay); ming.hammond@utah.edu (M. Hammond)

1 These authors contributed equally.
} 
The effects of spaceflight on plants have been studied since the 1960s on a variety of platforms and reviewed by Wolverton and Kiss (2009), Paul et al. (2013), Poulet et al. (2016), Vandenbrink and Kiss (2016), and Zabel et al. (2016). One key trend observed through several decades is the impacts of hardware design on biological results (Poulet et al., 2016; Zabel et al., 2016). Specifically, early experiments tended to lack ventilation, adequate volume, and advanced water and nutrient delivery systems seen on more modern platforms. Biological results from these early experiments often reported reduced growth and effects that could be traced to lack of convection such as hypoxic conditions in roots, reduced $\mathrm{CO}_{2}$ exchange on leaves, and buildup of volatile organic compounds (VOCs) including ethylene (Poulet et al., 2016). Modern platforms provide ventilation and environmental control which has improved plant growth in space (Poulet et al., 2016; Zabel et al., 2016).

Responses to microgravity are often species-specific and vary depending on the stage of development of the plant (Paul et al., 2013). For example, specific to Arabidopsis thaliana, phototropic response is more sensitive in $\mu \mathrm{G}$ than $1 \mathrm{G}$ and adequate to orient hypocotyls (Millar et al., 2010). Ferl et al. (2015) report differential expression for 1500 proteins for space-flown plants, and Correll et al. (2013) report differential transcription (two-fold difference) of 230 genes between space-flown and ground samples. Vandenbrink et al. (2019) report significant differential expression of 296 genes by RNA-seq.

Paul et al. (2005) report differential transcription (four-fold difference) of 182 genes including upregulation of some, but not all, heat shock genes even though growth temperatures were maintained below $25{ }^{\circ} \mathrm{C}$. Corroborating this finding, microgravity experiments in parabolic flight show increases of $0.9-1{ }^{\circ} \mathrm{C}$ in leaf surface temperature of sweet potato, barley, and wet paper replicas in microgravity (Kitaya et al., 2001, 2003). A transgenic strain of $A$. thaliana where green fluorescent protein was fused to genes of interest was flown to space and functioned as expected (Ferl and Paul, 2016). Though transgenic, the fluorescent reporter monitored native activity and was not an inducible expression system in the engineering context. There are several examples of similar systems with native promotors or gene fusions in the literature (Kittang et al., 2014; Paul et al., 2001, 2013b).

Towards the demonstration of on-demand gene expression via exogenous induction, we selected transgenic Arabidopsis thaliana pX7-AtPDSi that stably expresses a synthetic inducible RNAi gene silencing system (Guo et al., 2003) (Fig. 1). The synthetic biological circuit comprises the XVE activation system (Zuo et al., 2000) and CLX system (Zuo et al., 2001). The XVE expression system is a transcription factor-promoter pair that is activated by estradiol [21]. A PG10-90 constitutive promotor expresses an artificial transcription factor called XVE that consists of a fusion of particular domains from LaxA, VP16, and the human estrogen receptor. In the presence of estradiol, the XVE transcription factors bind to eight OLexA sites on the engineered DNA promoter, thereby activating production of the downstream gene of interest, which in this case is Cre recombinase from the CLX system.

Once it is produced, Cre will excise a portion of DNA between two loxP sites in the engineered CLX system, which conveniently provides a permanent change to the genomic DNA that can be analyzed post-flight via PCR. For the purpose of in-flight analysis, the DNA recombination event leads to production of an siRNA that silences the phytoene desaturase (PDS) gene. The result is a photobleached phenotype that is distinguishable through standard photographs. The XVE system was shown to yield $8 \mathrm{X}$ higher production levels than the commonly used strong constitutive promoter, CaMV 35S (Zuo et al., 2000) and be tightly regulated, having no leaky expression of the recombinase without estradiol present (Guo et al., 2003). Furthermore, the system could be activated at many stages of development and produce a spectrum of induction intensity.
One of the seminal discussions on the use of synthetic biology for space outlined several tasks that should be complete in a 5 yr timeframe (Langhoff et al. 2010). Among these were "testing synthetic biology techniques in microgravity", assessing the "viability and stability of [engineered] organisms in space", and the "development of RNAi technologies" (Langhoff et al. 2010). To the best of our knowledge, the experiment reported here represents the first space flown experiment to address any of these recommendations. The aim of this study is to demonstrate functionality of an exogenously inducible genetic circuit in plante during spaceflight as a first step towards enabling synthetic biology approaches for spaceflight. While transgenes with native promotors have been demonstrated in spaceflight, exogenous induction with small molecules, and a complex synthetic circuit employing non-native promotors (G10-90), non-native regulatory sites $\left(\mathrm{O}_{\text {LexA }}\right)$, non-native proteins (Cre and XVE fusion), and RNAi have not.

While we hypothesized that all of the biochemistry would be functional, this was not necessarily a given. Langhoff et al. 2010 suggest that synthetic constructs should be tested in space stating "since organisms may behave differently in space, ground research needs to be validated in the appropriate environments." Specifically, the lack of convection manifests itself typically by reducing chemical flux into and out of the organism and this could reduce the uptake of the estradiol induction molecule. This phenomenon has been shown to have major impacts on microorganisms (Benoit and Klaus 2007, Benoit et al. 2008, Zea et al. 2016). Additionally, the gene expression profile of the plant is radically different in the spaceflight environment. This could lead to interactions with the genetic circuit that differ from those observed on the ground. The flight demonstration provides flight heritage to a suite of genetic elements that can be incorporated into future designs with reduced risk (i.e. improved Technology Readiness Level). In the process a small hermetically-sealed plant growth module was developed and the design made openly available. The design is compatible with readily available commercial platforms on the International Space Station (ISS) such as the Space Application Services ICE Cubes platform and should facilitate future plant growth experiments in space, and on the surfaces of the Moon and Mars.

\section{Methods}

\subsection{Payload}

A sealed chamber of 0.631 served as the vessel which contained the experiment (Fig. 2). Seeds were fixed to filter paper using guar gum and hydraulically connected to the fluid delivery port through physical contact with a piece of gauze (Absorbent Cotton Co.). Media containing the inducer was stored in a spring- pressurized bag. Once commanded, a valve released the media to the gauze, which then diffused to the filter papers providing water and nutrients to the seeds. Growth lighting was continuously provided by blue and red LEDs at an intensity of 140-150 $\mu \mathrm{mol} \bullet \mathrm{m}^{-2} \bullet \mathrm{s}^{-1}$ following media delivery (specification sheet in supporting files). Images were taken every four hours in a sequence that consisted of turning off growth lights, turning on three white imaging lights, collecting an image then returning to the original growth light stage. The entire imaging sequence takes less than five seconds. Details of the payload, including design, testing, and operation, are included in the supporting files and should be adequate to produce a replicate of the flight hardware.

\subsection{Experimental setup}

\subsubsection{Growth chamber}

Whatman filter paper pieces $(17 \mathrm{CHR}, 70 \times 90 \mathrm{~mm})$ and aluminum combs were pre-measured and cut according to growth cassette slot dimensions. Seed placement was marked between comb sections with a pencil using the metal end of a mechanical pencil to create a pro- 

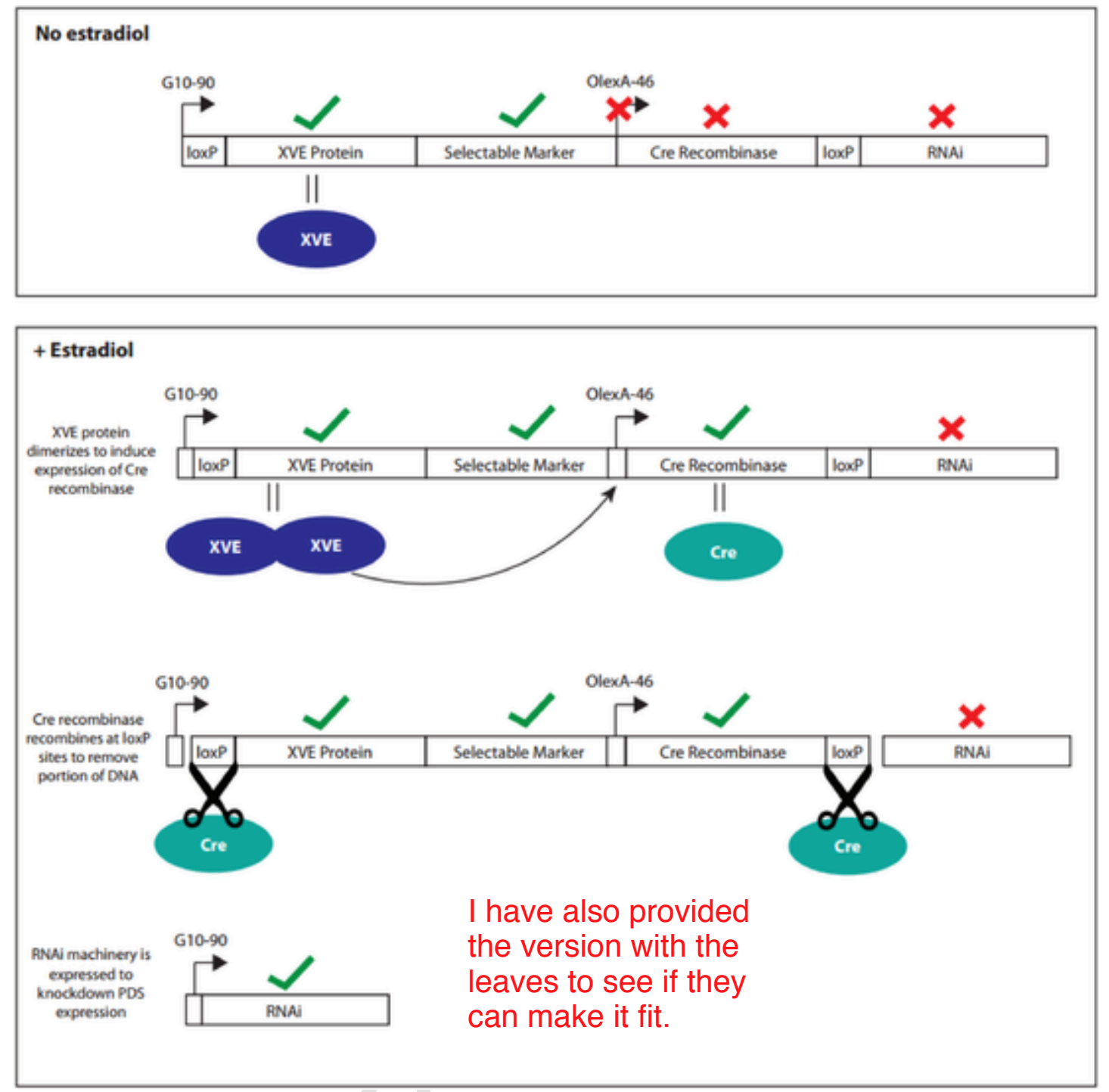

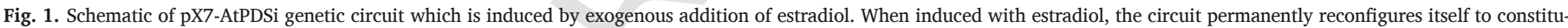

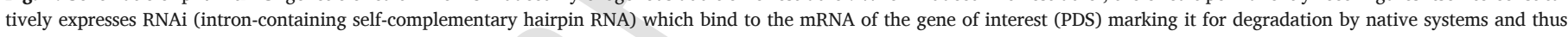

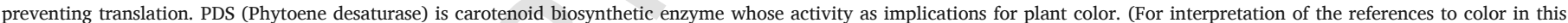
figure legend, the reader is referred to the web version of this article.)

nounced indent that could be viewed from the side. The growth cassette was assembled by filling each row with a "sandwich" of filter paper (seed markings), aluminum mesh, and filter paper (spacer, no seed markings). The seed markings were arranged to face the center of the cassette. A piece of folded gauze was placed at the bottom of an aluminum growth chamber. The gauze was centered to avoid contact with the edge of chamber and prevent blockage. Metal tweezers or forceps were used to grasp the filled growth cassette and lower it into the bottom of the chamber. The cassette was turned so that rows were aligned to suit the camera angle, and the cassette and gauze were pressed down to be flush with the bottom of the chamber.

The entire chamber was covered with aluminum foil and autoclaved at $121{ }^{\circ} \mathrm{C}$ or $134^{\circ} \mathrm{C}$. Once the cycle was complete, the exterior was wiped with $70 \%(\mathrm{v} / \mathrm{v})$ ethanol and then transferred to a sterile biohood. All metal tweezers and aluminum screws were autoclaved in a covered container or aluminum foil. Once the cycle was complete, materials were sprayed thoroughly with $70 \%$ ethanol and the container transferred to the biohood. All other materials were sprayed thoroughly with $70 \%$ ethanol and place in a biohood to dry under air flow for at least $30 \mathrm{~min}$. Electronic components were not autoclaved. Instead, these were sprayed with $70 \%$ ethanol and dried for at least $30 \mathrm{~min}$ (electronic components must be completely dry prior to assembly to avoid short-circuiting and equipment damage).

\subsubsection{Media/guar gum/seeds}

A $1 \% \mathrm{w} / \mathrm{v}$ guar gum solution was mixed in the biohood using the upper clear portion of the guar gum solution. A $50-100 \mathrm{~mL}$ container of 0.5X MS Salts, pH 5.8 was autoclaved and transferred to the biohood. Arabidopsis thaliana pX7-AtPDSi was kindly provided by Prof. Nam-Hai Chua (Rockefeller University) and Arabidopsis thaliana Col-0, the wild type strain, was originally obtained from Dr. Imara Perera (Department of Plant and Microbial Biology, North Carolina State University). Seeds were sterilized by five-minutes of hand swirling with $70 \%$ ethanol $+0.1 \%(\mathrm{v} / \mathrm{v})$ Tween-20 followed by double rinses with $100 \%$ ethanol and ten seconds of hand swirling. The seeds were then gently suspended in $100 \%$ ethanol and ejected onto an autoclaved Whatman filter paper 113 (125 mm Wet Strengthened Circles) to air dry completely. Sterile seeds were collected in autoclaved tubes and stored in a dark drawer until use. 

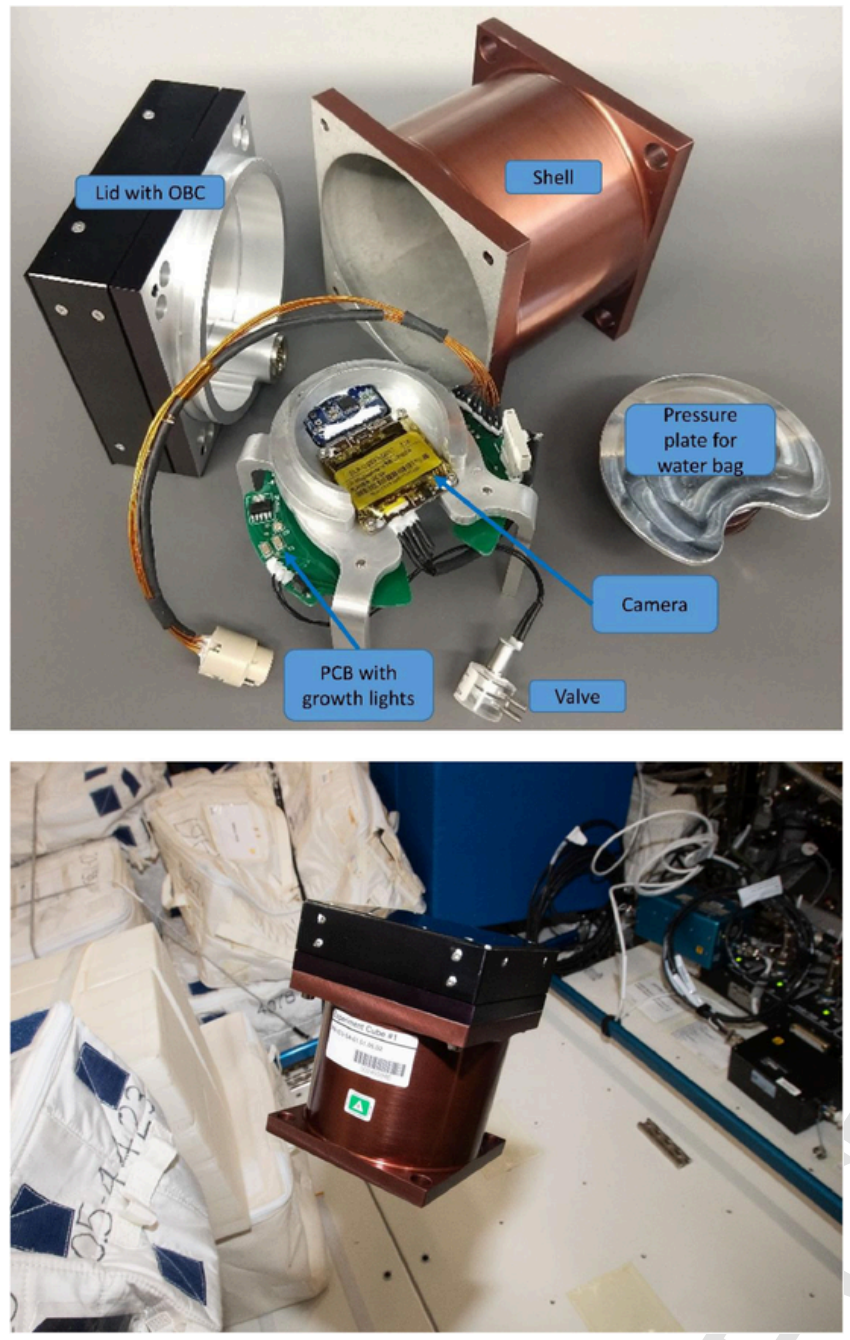

Fig. 2. (Top) diagram of the hardware unassembled. Water bag and seed cassette are not pictured. The PCB assembly is inserted into the shell after the cassette and followed by the pressure plate, water bag, and lid. (Bottom) space cube on ISS just before being placed in a bag and sealed for return to Earth.

\subsubsection{Assembly of growth chamber}

This assembly was performed in the biohood with pre-sterilized materials - the biohood was sprayed down with $70 \%$ ethanol and allowed to dry for at least $15 \mathrm{~min}$ before starting work. The autoclaved aluminum foil was also sprayed down and used as a work surface in the hood as a form of secondary containment. A sterile growth cassette was unwrapped in the hood and the blunt end of sterile tweezers was used to gently press each piece of filter paper down to ensure contact with the lower gauze layer. A small amount of seeds were tapped out of the tube (44 of each strain) into a sterile petri dish or onto a glass slide. A minimal amount of guar gum was added to coat the seeds. Forceps were used to gently place a seed within a pre-marked indent in filter paper. Seeds were placed in every indent, with pX7-AtPDSi seeds in the four rows closer to the water delivery opening (bottom of the images). A water indicator dot was added to the top edge of a piece of filter paper using forceps.

A total of $30 \mathrm{~mL}$ of $0.5 \mathrm{X}$ Murashige and Skoog Salts (see supplemental) was poured into a sterile $50 \mathrm{~mL}$ Falcon tube. $30 \mu \mathrm{L}$ of $2 \mathrm{mM}$ estradiol in ethanol (1:1000 dilution) was added for a final concentration of $2 \mu \mathrm{M}$ estradiol in 0.5X MS salts. Prior to flight, estradiol was found to have minimal degradation after storage at room temperature for 30 days [Kitto, 2019]. A sterile syringe + needle (18 G) was used to fill the media bag with $28 \mathrm{~mL}$ media. Then the cube was assembled.

\subsection{Flight}

Two payloads were prepared: one for flight (flight cube) and one to serve as the non-flight control (ground cube). Preparation occurred in Utah and the flight unit was mailed to the launch site without X-ray. In total, the cubes were stored for eight days prior to launch. Activation of the experiment occurred nine days after launch and the duration of the experiment was twenty days (Fig. 3). The ground cube was prepared and operated with a one-day delay compared to the flight cube. This allowed time to respond to events as they occurred in flight cube. Otherwise, the cubes were set up using identical batches of sterilized seeds and growth media. Fresh estradiol stocks were prepared and the hardware was assembled as described above.

Occasionally the power to a cube was cycled off then back on for operational reasons. Power cycling causes the unit to reboot and desynchronizes the four-hour imaging sequence. Also, the first few images taken after rebooting are overexposed and washed out and could not be used in analysis. These images were removed from analysis. Power cycles occurred on Day 8 for the ground cube and Days 7 and 15 for the flight cube.

At the end of the active phase, units were powered down removing light to the plants and stored at $4 \mathrm{C}$ until analysis. The space unit returned to the USA and was delivered as-flown without x-ray to France for post flight analysis. The ground unit was disassembled in Utah, biomass flash frozen and mailed to France via normal post (with x-ray) on dry ice.

\subsection{Image analysis}

For quantitative analysis, every fourth image (taken approximately every eight hours) from the flight set and the closest matching image in the ground set were analyzed through an image analysis process (supporting files). Each image was $2592 \times 1944$ pixels. First, plants were traced and cut from the original images then pasted into a blank pure white image such that only plants were present in the new image used for calculations. This was done manually in paint.net 4.1 .5 by a single person for consistency. The file format used follows that of the original image, which was a 24-bit Portable Network Graphics (PNG), where each of three channels has eight bits (0-255). The leaf area in each image is estimated by counting the number of plant pixels in the image.

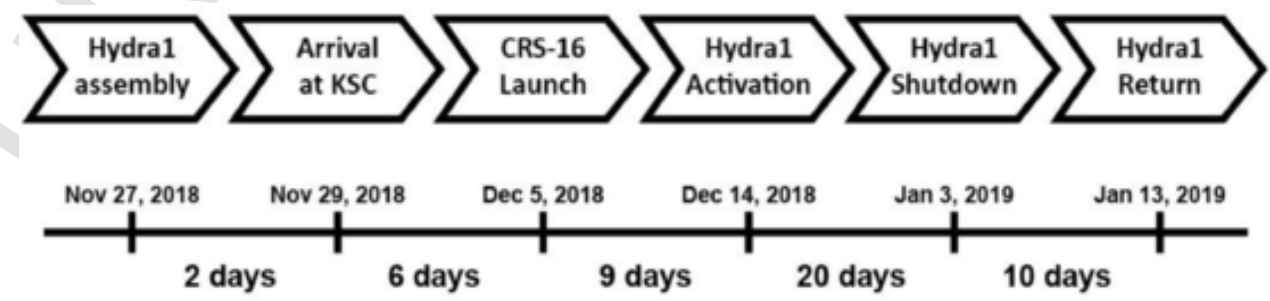

Fig. 3. Experimental timeline of the space cube. The cube was active on orbit for twenty days. The ground cube was operated on a one-day delay. 
Plant pixels are defined as those pixels that are not pure white. The genetic circuit is designed to induce photobleaching in plant leaves that is observable visibly as a whitening of what would otherwise be green leaves. Thus, the effectiveness of the genetic circuit can be evaluated by quantifying the greenness of the plant leaves. The greenness of each pixel was calculated as green/(green + blue + red) where green, blue, and red are the bit values for the corresponding channels. The index reported is the median value for all pixels in a sample. Other indexes from the literature were also calculated but there were no major differences in the conclusions drawn. Two controls were conducted to verify that the greenness was not an artifact of the image itself or lighting conditions. Sections of the white filter paper and aluminum cassette were processed in a similar manner to the plants for comparative purposes.

\subsection{Polymerase chain reaction}

A polymerase chain reaction (PCR) can be used to verify the production and activity of Cre recombinase protein. Specifically, primer pairs designed to amplify around the excised portion will produce smaller fragments while primer pairs designed to amplify across a LoxP site will produce no fragments from DNA template that has successfully undergone CLX activation. Template DNA was isolated from whole plant extracts using a Qiagen DNeasy Plant Mini Kit according to manufacturer's instructions. PCR reactions were carried out following the methods and primer pairs of Guo et al. (2003). PCR reactions were separated by gel electrophoresis and imaged to determine the size of the amplified bands. Visible bands were also physically extracted, cleaned (using a Macherey-Nagel Nuceo-Spin PCR clean-up kit according to manufacturer's instructions), and Sanger sequenced at the IBMP "Gene Expression Analysis" platform using an ABI3130 XL sequencer, to further compare them to the expected results or identify their origin.

\section{Results and discussion}

\subsection{Establishment of experimental conditions}

The experimental conditions that needed to be adapted for the launch and flight conditions are summarized here (for a detailed account, see ref. Kitto, 2019). Important considerations included multiday storage of prepared seeds and reagents at or above room temperature, which could lead to growth of biological contaminants, loss of activity due to reagent decomposition (e.g. the inducer estradiol), and loss of seed viability and germination efficiency due to storage conditions and/or exposure to VOCs and leached chemicals from growth chamber materials. For example, instead of published conditions for seed germination on phytoagar plates (1x MS salts with $3 \%$ sucrose, $0.8 \%$ agar, $2 \mu \mathrm{M}$ estradiol), seeds were germinated and grown affixed to filter paper with $0.5 \mathrm{X}$ MS salts with no sucrose, $2 \mu \mathrm{M}$ estradiol. Sucrose was removed because while it greatly enhances plant growth, it led to high incidence of biological contamination even with rigorous sterilization techniques. It was found that $0.5 \mathrm{X}$ MS salts was needed to promote seed germination and growth, as $2 \mu \mathrm{M}$ of the inducer estradiol was toxic to both wildtype and transgenic seeds germinated with water alone. For this reason, the higher concentration of estradiol $(10 \mu \mathrm{M})$ was not used, and although some decomposition of the inducer was expected under these conditions, we established in ground tests that aged seeds with aged $2 \mu \mathrm{M}$ estradiol ( $30 \mathrm{~d}$ post-sterilization and post-stock preparation, respectively) still gave visible photobleaching.

The number of wildtype and transgenic seeds were chosen to provide adequate statistical sampling while permitting growth for enough days to visualize germination, growth, and photobleaching before depletion of $\mathrm{CO}_{2}$ in the hermetically sealed chamber limited all plant growth. Tests were conducted to measure and adjust the light intensity of the LED light array to enable high germination rates and normal plant growth. Growth chamber materials were selected based on biocompatibility tests performed with seeds germinated with growth media containing $2 \mu \mathrm{M}$ estradiol and off-gassed as time permitted to reduce the exposure of the seeds or plants to VOCs.

Other conditions that could affect biological samples in general during launch include vibration and g-forces. Thus, it was beneficial to store and launch seeds rather than pre-germinate and launch plant seedlings. In summary, these results highlight that even robust synthetic biological circuits developed for the laboratory setting may require, at minimum, adjustment or re-optimization of experimental procedures to work after prolonged storage and launch, in addition to spaceflight conditions.

\subsection{Germination and growth}

Images were reviewed to detect emergence of radicle, hypocotyl and cotyledon. Germination yields were higher in the ground cube than the space cube and in both cubes transgenic seeds had higher germination yields than wild type seeds (Table 1). With the exception of pX7-AtPDSi in the ground cube, germination yields were lower than the $80 \%$ threshold which is common for Col-0 (Rivero et al., 2014). In general, the resolution of the images was not adequate to distinguish between the specific parts but was adequate to detect activity. The green color of the cotyledon was more obvious against the white filter paper so the detected activity is likely weighted toward cotyledon emergence. The average time to first activity detected for each seed followed similar trends to the germination yield with ground seeds germinating sooner than space ones and transgenic seeds germinating sooner than wild type. Boyes et al. (2001) report an average of $4.3+/-0.4$ days (mean $+/$ - SD) for radicle emergence and $5.5+/-0.6$ days for hypocotyl and cotyledon emergence in Col-0 while Rivero et al. (2014) report germination of 3-5 days under continuous lighting for Arabidopsis in general. The seeds in the ground cube match these values well but the space flown seeds are delayed. Further, there was substantial variability in activity timing. CVs for all but the Col-0 flight seeds ranged between 22 and 28\%, which is more than twice the 10.3-11.2 reported by Boyes et al. (2001). In the flight cube, Col-0 seeds germinated as late as 16.75 days which is twice as late as in the ground cube. The CV of 54\% reflects this.

Given the sealed nature of the cube, a reduction in germination could lead to higher biomass yields per plant, but this is the opposite of what was observed. Estimates of the leaf area from pixel counts of photographed leaves, shows that plants in the ground cube were an order of magnitude larger than their space-flown counterparts (Fig. 4). In the flight cube, leaf area decreases starting at Day 9 in pX7-AtPDSi and Day 13-14 in Col-0 indicating that the growth had halted. In both conditions, the wild type strain yielded higher leaf area despite lower germi-

Table 1

Germination yield and general statistics of first activity timing.

\begin{tabular}{|c|c|c|c|c|}
\hline & \multicolumn{2}{|l|}{ Flight } & \multicolumn{2}{|l|}{ Ground } \\
\hline & Col-0 & pX7-AtPDSi & Col-0 & pX7-AtPDSi \\
\hline Geminated/total & $22 / 43$ & $26 / 40$ & $25 / 43$ & $41 / 43$ \\
\hline Gemination Yield (\%) & 0.51 & 0.65 & 0.58 & 0.93 \\
\hline Mean (d) ${ }^{* *}$ & 6.66 & 5.69 & 4.39 & 3.43 \\
\hline Std Err of Mean (d) ${ }^{* *}$ & 0.55 & 0.21 & 0.19 & 0.12 \\
\hline Std Dev (d) ${ }^{* *}$ & 3.60 & 1.30 & 1.25 & 0.80 \\
\hline $\mathrm{CV}(\%)^{* *}$ & 0.54 & 0.23 & 0.28 & 0.23 \\
\hline $\operatorname{Min}(d)^{* *}$ & 2.67 & 3.17 & 3.00 & 2.33 \\
\hline $\operatorname{Max}(d)^{* *}$ & 16.75 & 7.50 & 7.67 & 6.00 \\
\hline Range (d) ** & 14.08 & 4.33 & 4.67 & 3.67 \\
\hline
\end{tabular}

** time to first detectable activity in images. 

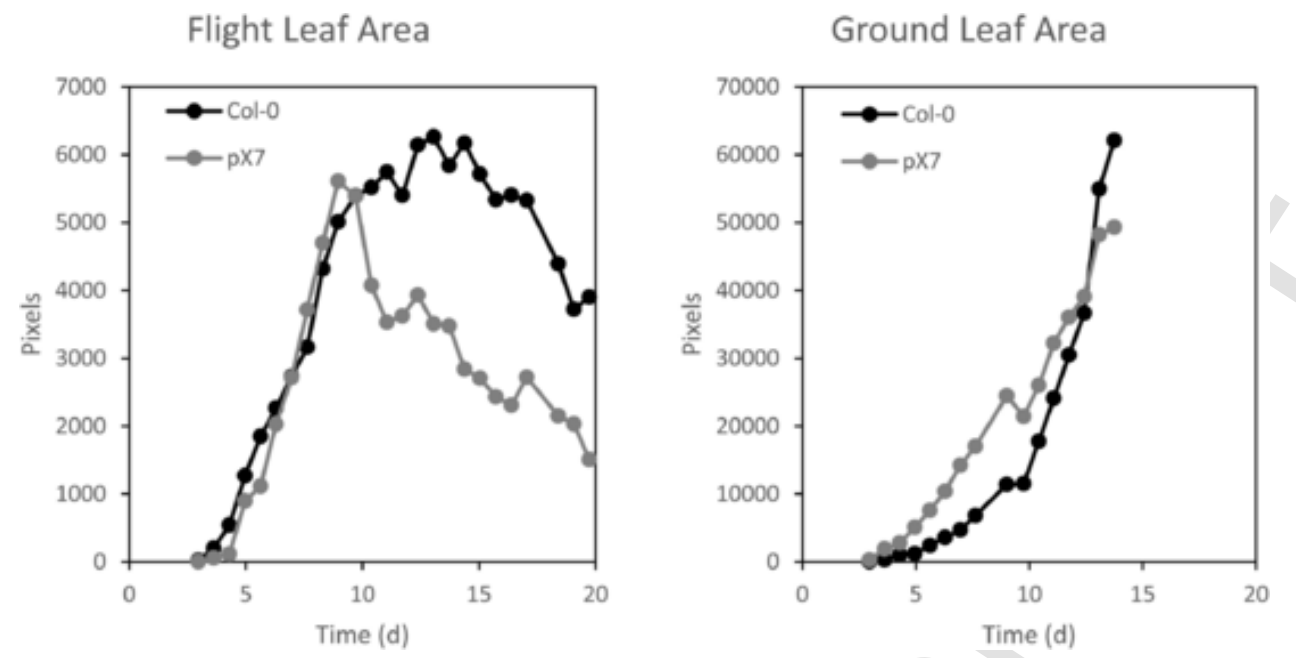

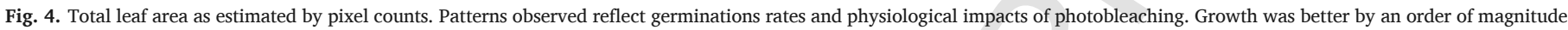
in the ground cube than the space cube indicating stresses in the flight cube.

nation rates. For the transgenic strain, the reduction in leaf area is a result of leaf photobleaching, which is known to detrimentally affect plant growth. In some pX7-AtPDSi plants, later leaves do not photobleach likely because the estradiol has been depleted by the point these leaves are produced. This observation was also reported by Guo et al. (2003) in lab experimental conditions. Taken together, the reduced growth compared to Col-0 support that the transgenic plants have reduced photosynthesis due to photobleaching. The visible difference between some early and later transgenic leaves observed in the ground cube support that estradiol is required to induce protein production (e.g. of Cre recombinase), as its depletion during the experiment leads to later leaves not being photobleached. Due to the reduced growth of plants in the flight cube, later leaves were not visible, but we expect that estradiol would be depleted at a similar or accelerated rate (see Temperature results).

\subsection{Photobleaching}

Visual inspection of the images confirms photobleached leaves and thus activation of the pX7-AtPDSi system in both ground and flight conditions (Fig. 5, Supplementary Files 1 and 2). The ground Col-0 plants are distinctly green while the flight Col-0 plants are more ambiguous. Quantitative image analysis however confirms that Col-0 leaves are greener than their pX7-AtPDSi counterparts in both conditions (Fig. 6). At day 9 in both conditions, photobleaching starts and leads to a negative slope in the leaf area that arises from arrested growth. The onset of photobleaching leads to a distinct cross in the greenness profiles at day 10. Furthermore, in both conditions, the maximum difference in the greenness occurs at day 14-15. These temporal similarities between the flight and ground cubes indicate the pX7-AtPDSi system functioned similarly despite large differences in the plants themselves.

For both the flight and ground units, the background greenness was nearly static throughout the experiment (Supplementary Figure 1). Some peaks occur where power was cycled, causing the first few photos to be exposed differently. This was a known issue prior to flight. There is a small bias between the wildtype and transgenic halves of the cube but the magnitude of 0.004 is $\sim 6 \%$ of the observed range and within the accuracy of the methods employed.

In general, the plants in the ground cube were greener than those in the flight cube, with the final days of the experiment showing the most difference. The flight cube exhibits a general decrease in greenness starting at Day 11 in the Col-0 plants and Day 6 in pX7-AtPDSi. In the
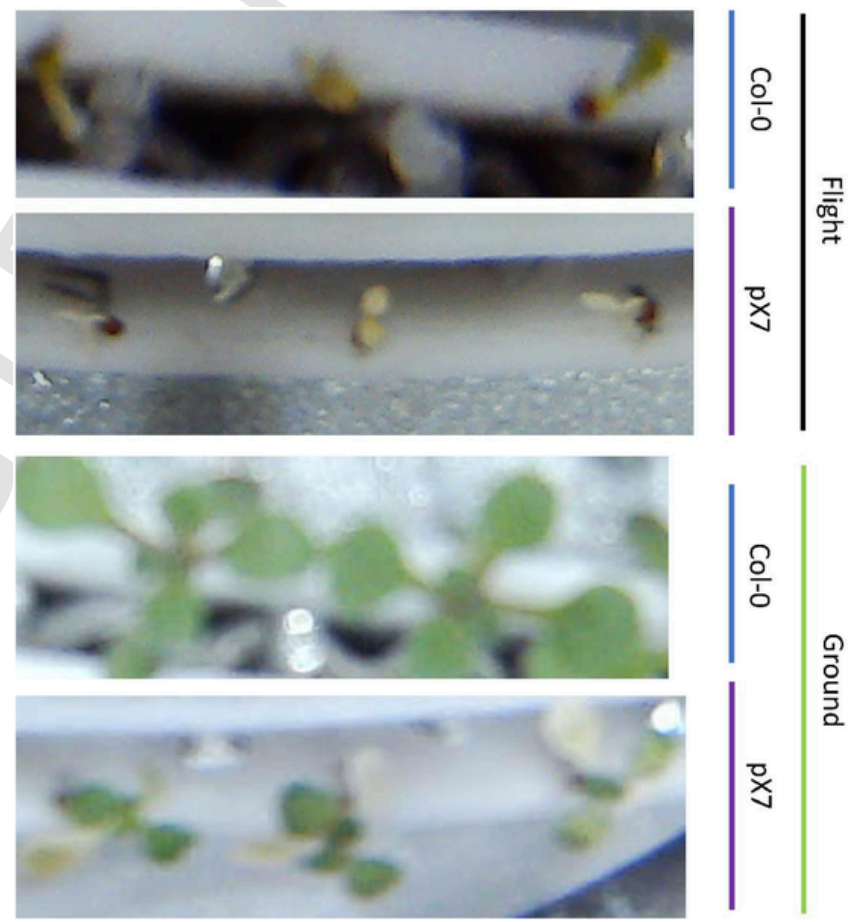

Fig. 5. Images of plants from the experiment: top two rows are flight plants (Day 15), bottom two rows are ground plants (Day 14). The flight plants were smaller than the ground plants indicating stresses from spaceflight. In both flight and ground cubes, the pX7-AtPDSi plants photobleached while the Col-0 plants did not. In the ground cube, pX7-AtPDSi plants produced green leaves later in the experiment as estradiol had been consumed. Time lapse movies are given in Supplementary Files 1 and 2 and all images are made publicly available. (For interpretation of the references to color in this figure legend, the reader is referred to the web version of this article.)

ground cube, pX7-AtPDSi plants are generally static in greenness while the Col-0 plants are increasing throughout the experiment. Once the induction molecule becomes depleted, new leaves added by pX7-AtPDSi plants are green rather than photobleached (Supplementary File 1) (Guo et al., 2003). Both early and later leaves were included in the quantitative analysis to avoid user bias, and the greenness index analysis reports the median of analyzed pixels. Thus the flattening greenness in the ground condition pX7-AtPDSi plants reflects a bimodal distribution that is confirmed by histograms (Supporting Files). Nevertheless, the observation of photobleaching visually and with quantitative analy- 
Flight Greenness Index

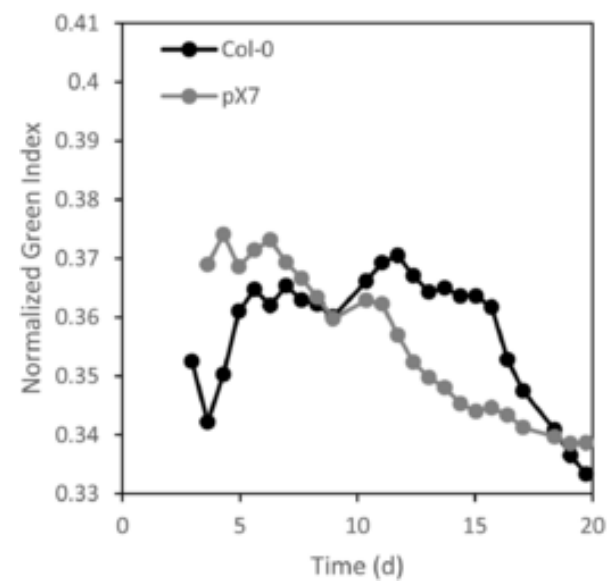

Ground Greenness Index

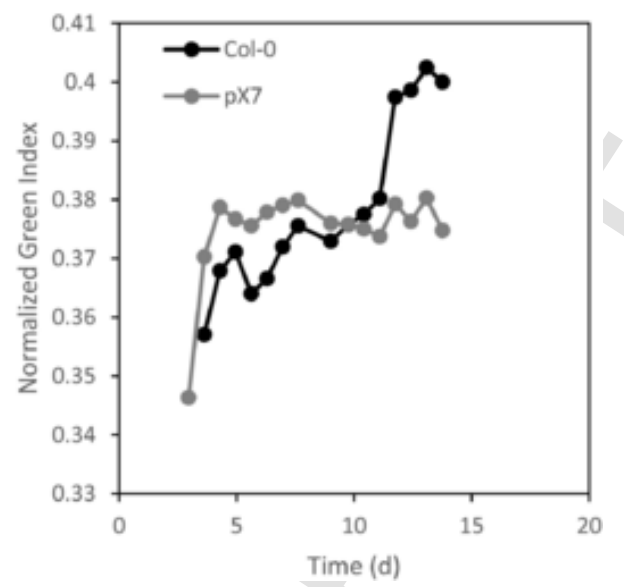

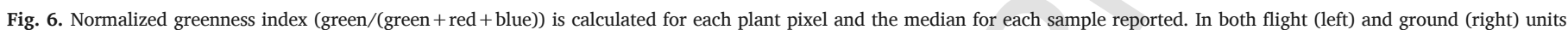
transgenic plants start greener but flip at about Day 9. (For interpretation of the references to color in this figure legend, the reader is referred to the web version of this article.)

sis in the transgenic plants provides support that Cre recombinase production and siRNA activity were induced during spaceflight, as both functions were necessary to lead to the photobleaching phenotype. One challenge of the study is the hardware constraint of a single fluid delivery to all plants. Because of this, a key control treatment of non-induced pX7-AtPDSi plants was not conducted and thus it is possible, though, unlikely, that spaceflight alone induced the pX7-AtPDSi system.

\subsection{Polymerase chain reaction}

PCR reactions can be used to verify functionality of the CLX element because it relies on excision of a portion of DNA (Fig. 7) (Guo et al., 2003). Biomass for all Col-0 and pX7-AtPDSi plants were consolidated due to the limited quantity and quality (due to time delays and room temperature storage) of the biomass that could be obtained after the experiment. None of the Col-0 wild type reactions produced PCR bands confirming that they do not have the transgenic elements of pX7-AtPDSi. As expected for a functional pX7-AtPDSi system, there is a $992 \mathrm{bp}$ band in the P1/P4 lane of both the flight and ground samples. However, there is also a 696 bp band in the P1/P2 lane and $1331 \mathrm{bp}$ band in the P3/P4 lane in both the flight and ground samples, indicating that some DNA has not undergone Cre/LoxP recombination. For both space-flown and ground samples, the intensity of the P1/P4 pair band is similar to that of the P1/P2 pair while the P3/P4 band is much darker, indicating similar behavior between the samples. Guo et al. (2003) also report P3/P4 bands that have higher intensity than the P1/P2 bands. This intensity pattern is likely an artifact of the primer pair and not indicative of the biochemical functionality. The P1/P2 and P3/P4 bands in the ground sample can be explained by the later stage leaves, which were produced after estradiol had been consumed (Guo et al., 2003). The same explanation could apply to the flight sample,
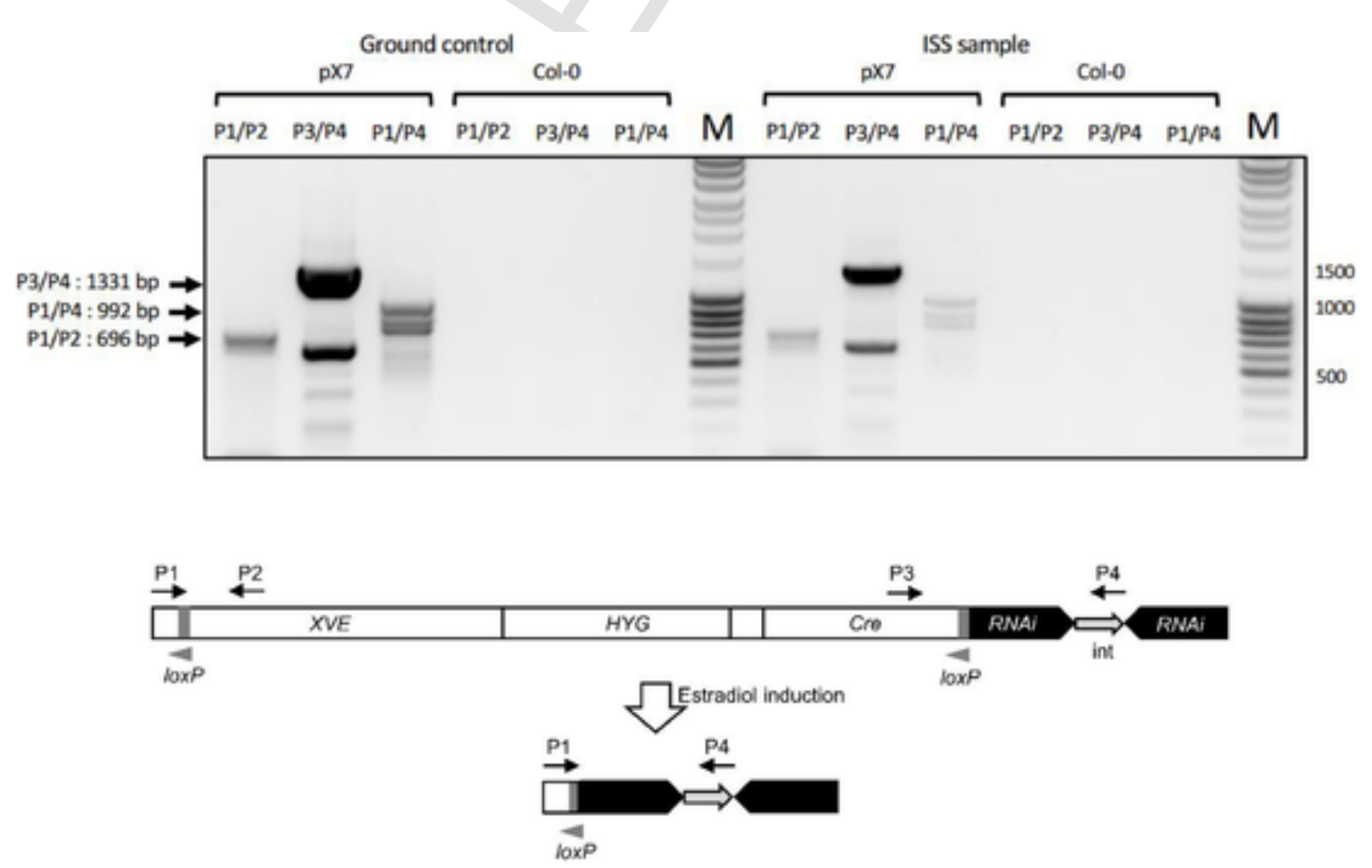

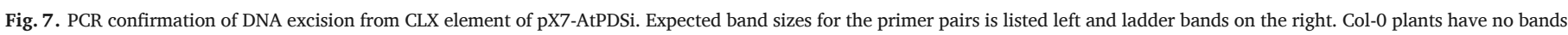
because the transgenic elements are not present. The P1/P4 lane confirms successful excision. Smaller bands in the P3/P4 lane were due to contamination. 
although later stage leaves were not visible, or alternatively the induction of the pX7-AtPDSi system was partial, which could be due to flight conditions affecting the plants and/or increasing estradiol breakdown.

In both the space and ground samples, beyond the 992 bp bands in the P1/P4 lane, other bands of smaller molecular weights can also be detected. These amplifications cannot be explained by the pX7-AtPDSi system. The different bands were excised from gel and analyzed by Sanger sequencing. This revealed that the $992 \mathrm{bp}$ fragments indeed correspond to the recombination product expected from the pX7-AtPDSi system, while the lower molecular weight bands in the P1/P4 lanes are off-target amplifications of a root bacteria Mesorhizobium spp genomic sequence. This contaminating prokaryote was likely present in Arabidopsis seed batches used here, as classically observed, and its genomic DNA was extracted together with plant genomic DNA (Lundberg et al., 2012).

\subsection{Temperature}

Each cube contained a thermometer on the internal circuit board which was used to record temperature approximately every two minutes. The average temperature reported by the flight cube was $34.29^{\circ} \mathrm{C}$ while the average temperature in the ground cube was $29.13^{\circ} \mathrm{C}$. The lack of convection in the space environment can cause differences in the circuit board temperature that do not necessarily correspond to differences in temperature at the plants. Furthermore, small differences in coating thicknesses could impact the thermal distribution on the circuit boards. After the flight, a calibration curve was produced in the flight cube to connect outside temperatures to the thermometer output. This suggests that the flight cube was likely operating at an external temperature $26-27{ }^{\circ} \mathrm{C}$ compared to $23-25^{\circ} \mathrm{C}$ in the ground cube. The temperature range for $A$. thaliana growth is $16-25^{\circ} \mathrm{C}$ with younger plants (pre early rosette stage) being particularly sensitive to higher temperatures (Rivero et al., 2014). Paul et al. (2005) reported overexpression of heat shock genes in space flown $A$. thaliana with temperatures in the range reported here.

\section{Conclusions}

Despite the interest in synthetic biology for space applications, very little, if any at all, has been reported about synthetic biology deployment in the actual space environment. As synthetic biology functions within the context of an organism, the organism's microgravity phenotype is paramount to a successful synthetic biology output. In this experiment, an in plante synthetic biological circuit was evaluated in spaceflight. The circuit was designed to produce a protein (Cre recombinase) and an siRNA whose activities in sequence lead to leaf photobleaching for easy visualization and measurement on orbit. Visual inspection of the images illustrates that pX7-AtPDSi leaves are lighter than their wild type Col-0 counterparts. This is despite challenges with plant growth on orbit. Calculating the greenness index enables quantitative analysis and helps to remove bias. The greenness index confirms that pX7-AtPDSi plants were less green than their Col-0 counterparts. Furthermore, the temporal patterns in greenness index are similar between the flight and ground cubes. Finally, since Cre causes a DNA recombination event, PCR after sample retrieval could be used to confirm at a molecular level that the pX7-AtPDSi system functioned in space as it did on the ground.

The differences in germination and growth patterns between the flight and space cubes can be explained by higher than optimal temperatures in the space cube. This experiment was among a trial batch intended to test the newly-installed ICE Cubes platform on the Columbus module of the ISS. As such, detailed thermal profiles of the ICE Cubes platform on orbit were not available prior to flight. A thermometer installed on the internal circuit board indicates that the external temperature - thus the temperature most closely experienced by the plants - was $26-27^{\circ} \mathrm{C}$. This is $1-2{ }^{\circ} \mathrm{C}$ above $A$. thaliana's optimum growth range. Rivero et al., 2014 report negative responses particularly through germination and early rosette stages when exposing A. thaliana to temperatures above $25^{\circ} \mathrm{C}$. Microgravity further compounds the overheating issue by reducing the plants' ability to regulate their temperature lower (Kitaya et al., 2001, 2003) due to a lack of convection and others have reported over expression of heat shock proteins in space flown $A$. thaliana (Paul et al., 2005).

Another environmental stress may have been high humidity. While the humidity was not measured, condensation visible on the walls and cassette are consistent with a high relative humidity as would be expected in a sealed chamber. The humidity would likely be similar in both cubes so does not explain the difference in germination yields or growth but may serve to push the plants into a stressed state where other stresses arising from spaceflight could act more prominently. Rivero et al., 2014 report seed sterility can result from humidity over 90\%. Despite the combinatorial stresses from spaceflight and higher than optimal temperatures, the synthetic biological circuit was still functional, demonstrating the circuit's robustness.

Demonstrating a functional synthetic biological circuit in the spaceflight environment is the first step toward development of synthetic biology applications for spaceflight. The pX7-AtPDSi system has several unique aspects that each had to function in order to obtain the observed result like exogenous induction with a small molecule and non-native regulatory elements. Specifically, the XVE estradiol-inducible transcription factor, Cre/LoxP recombination system, and RNAi post-transcriptional gene silencing must have functioned as subcomponents to the combined pX7-AtPDSi system. XVE can be used to control level and timing of expression of any gene of interest in response to the inducer estradiol, while RNAi can be used to repress expression of any target gene(s). Cre/LoxP can be used to up regulate, down regulate, delete, exchange, and reverse genes and DNA segments which provides many design opportunities when combined with a tightly regulated activator. Despite loss of convection which could impact small molecule uptake and modified gene expression profiles resulting from spaceflight, all of these biological control circuits appear to have functioned as intended though suboptimal growth hinders a more conclusive determination. Synthetic biology projects intended for space can incorporate these elements in their designs to mitigate the risk of unknown behaviors during spaceflight. Future studies should verify that spaceflight alone, without chemical induction, does not induce the circuit.

In order to facilitate future plant studies in space, the design developed as part of the experiment has been made open and available (Supplemental online material. The cost to construct the payload is less than 10,000 USD dominated by the cost of the machining and hermetic seal. In-house machining could bring the unit cost down to $\sim 2000$ USD. Space Applications Services and other providers are advertising flights for less than 70,000 USD, which is an attainable price point for many academic and research groups. Further, the design now has flight heritage reducing the risk for future flights.

The experiment reported here is an early example of synthetic biological elements being tested in space. As the biological elements must work within organisms, context is an important consideration during design. Spaceflight is known to produce different phenotypes so working with space flown elements is ideal. Alternatively, new elements can be tested in the spaceflight environment using an open design.

\section{Uncited references}

$$
\text { , }
$$

\section{Author contributions}

Conceptualization: RK, YD, JB, MFH, RB, JJF, BO, CPM, CW, MCH. Methodology: RK, YD, JB, MFH. Formal analysis: JB, YD, MM, PG, LD, $\mathrm{DH}, \mathrm{CV}, \mathrm{NC}, \mathrm{MA}$. Writing- original draft: JB, RK, YD, MCH, CPM. All 
co-authors reviewed and approved the final draft.

\section{Declaration of Competing Interest}

The authors declare that they have no known competing financial interests or personal relationships that could have appeared to influence the work reported in this paper.

\section{Funding}

Funding at NASA Ames was provided by the Ames Center Innovation Fund. Work at UC Berkeley and University of Utah was funded by BWF CASI award 1007224 (to MCH), NIH P50 GM102706 (to Center for RNA Systems Biology), and University of Utah (to MCH). ISU received funding from Eurometropole (the City of Strasbourg) and Groupe des Assurances Mutuelles Agricoles of Strasbourg (Groupama). The spaceflight was provided by Space Applications Services.

\section{Supplementary materials}

Supplementary material associated with this article can be found, in the online version, at doi:10.1016/j.1ssr.2020.09.002.

\section{References}

Boyes, D., Zayed, A., Ascenzi, R., McCaskill, A., Hoffman, N., Davis, K., Görlach, J., 2001. Growth Stage-based phenotypic analysis of arabidopsis: a model for high throughput functional genomics in plants. Plant Cell 13, 1499-1510.

Cesaretti, G., Dini, E., DeKestelier, X., Colla, V., Pambaguian, L., 2014. Building components for an outpost on the Lunar soil by means of a novel 3D printing technology. Act. Astronautica 93, 430-450.

Correll, M., Pyle, T., Millar, K., Sun, Y., Yao, J., Edelmann, R., Kiss, J., 2013. Transcriptome analyses of Arabidopsis thaliana seedlings grown in space: implications for gravity-responsive genes. Planta 238, 519-533.

Du, B., Daniels, V., Vaksman, Z., Boyd, J., Crady, C., Putcha, L., 2011. Evaluation of Physical and Chemical Changes in Pharmaceuticals Flown on Space Missions. Am. Assoc. Pharmaceut. Sci. 13 (2), 299-308.

Ferl, R., Koh, J., Denison, F., Paul, A.-.L., 2015. Spaceflight induces specific alterations in the proteomes of Arabidopsis. Astrobiology 15 (1), 32-56.

Ferl, R., Paul, A.-.L., 2016. The effect of spaceflight on the gravity-sensing auxin gradient of roots: GFP reporter gene microscopy on orbit. NPJ Microgravity 15023.

Guo, H.-.S., Fei, J.-.F., Xie, Q., Chua, N.-.H., 2003. A chemical-regulated inducible RNAi system in plants. Plant J. 34, 383-392.

Kitaya, Y., Kawai, K., Tsuruyama, J., Takahashi, H., Tani, A., Goto, E., Saito, T., M, Kiyota, 2001. The effect of gravity on surface temperatures of plant leaves. Adv. Space Res. 28 (4), 659-664.

Kitaya, Y., Kawai, K., Tsuruyama, J., Takahashi, H., Tani, A., Goto, E., Saito, T., Kiyota, M., 2003. The effect of gravity on surface temperatures of plant leaves. Plant Cell Environ. 26, 497-503.
Kittang, A.I., Iversen, T.H., Fossum, K.R., Mazars, C., Carnero-Diaz, E., Boucheron-Dubuisson, E., Le Disquet, I., Legue, V., Herranz, R., Pereda-Loth, V., Medina, F., 2014. Exploration of plant growth and development using the European Modular Cultivation System facility on the International Space Station. Plant Biol. 16, $528-538$.

Kitto, R., 2019. Evaluating RNA-Based Gene Regulation Across Different Systems. University of California dissertation.

Llorente, B., Williams, T., Goold, H., 2018. The multiplanetary future of plant synthetic biology. Genes. 9 (348).

Lundberg, D., Lebeis, S., Paredes, S., Yourstone, S., Gehring, J., Malfatti, S., Tremblay, J., Engelbrektson, A., Kunin, V., Glavina del Rio, T., Edgar, R., Eickhorst, T., Ley, R., Hugenholtz, P., Tringe, S., Dangl, J., 2012. Defining the core Arabidopsis thaliana root microbiome. Nature 488, 86-90.

Menezes, A., Cumbers, J., Hogan, J., Arkin, A., 2015. Towards synthetic biological approaches to resource utilization on space missions. J. R. Soc. Interface 12, 20140715.

Millar, K., Kumar, P., Correll, M., Mullen, J., Hangarter, R., Edelmann, R., Kiss, J., 2010. A novel phototropic response to red light is revealed in microgravity. New Phytol. 186, $648-656$.

Paul, A.-.L., Daugherty, C.J., Bihn, E.A., Chapman, D.K., Norwood, K.L., Ferl, R.J., 2001. Transgene expression patterns indicate that spaceflight affects stress signal perception and transduction in arabidopsis. Plant Physiol. 126, 613-621.

Paul, A.-.L., Poop, M., Gurley, W., Guy, C., Norwood, K., Ferl, R., 2005. Arabidopsis gene expression patterns are altered during spaceflight. Adv. Space Res. 36, 1175-1181.

Paul, A.-.S., Wheeler, R., Levine, H., Ferl, R., 2013. Fundamental pant biology enabled by the Space Shuttle. Am. J. Bot. 100 (1), 226-234.

Paul, A.-L., Zupanska, A.K., Schultz, E.R., Ferl, R.J., 2013. Organ-specific remodeling of the Arabidopsis transcriptome in response to spaceflight. BMC Plant Biol. 13, 112.

Poulet, L., Fontaine, J., Dussap, C., 2016. Plant's response to space environment: a comprehensive review including mechanistic modelling for future space gardeners. Botany Lett. 163 (3), 337-347.

Ranneklev, S., Baath, E., 2003. Use of phospholipid fatty acids to detect previous self-heating events in stored peat. Appl. Environ. Microbiol. 69 (6), 3532-3539.

Rivero, L., Scholl, R., Holomuzki, N., Crist, D., Grotewold, E., Brkljacic, J., 2014. Handling arabidopsis plants: growth, preservation of seeds, transformation, and genetic crosses. Arabidopsis Protocols Methods Mole. Biol. 1062, 3-25.

Vandenbrink, J., Kiss, J., 2016. Space, the final frontier: a critical review of recent experiments performed in microgravity. Plant Sci. 243, 115-119.

Vandenbrink, J., Herranz, R., Poehlman, W., Feltus, F., Villacampa, A., Ciska, M., Medina, F., Kiss, J., 2019. RNAseq analyses of Arabidopsis thaliana seedlings after exposure to blue-light phototropic stimuli in microgravity. Am. J. Bot. 106, 1466-1476.

Villette, C., Maurer, L., Delecolle, J., Zumsteg, J., Ehrardt, M., Heintz, D., 2019. In situ localization of micropollutants and associated stress response in Populus nigra leaves. Environ. Int. 126, 523-532.

Wolverton, C., Kiss, J., 2009. An update on plant space biology. Gravit. Space Biol. 22 (2), 13-20.

Wong, J., Pfahnl, A., 2014. 3D printing of surgical instruments for long-duration space missions. Aviat. Space Environ. Med. 85 (7), 758-763.

Zabel, P., Bamsey, M., Schubert, D., Tajmar, M., 2016. Review and analysis of over 40 years of space plant growth systems. Life Sci. Space Res. (Amst) 10, 1-16.

Zhang, B., Shanmugaraj, B., Daniell, H., 2017. Expression and functional evaluation of biopharmaceuticals made in plant chloroplasts. Curr. Opin. Chem. Biol. 38, 17-23.

Zuo, J., Niu, Q.-.W., Chua, N.-.H., 2000. An estrogen receptor-based transactivator XVE mediates highly inducible gene expression in transgenic plants. Plant J. 24 (2), 265-273.

Zuo, J., Niu, Q.-.W., Moller, S., Chua, N.-.H., 2001. Chemical-regulated, site-specific DNA excision in transgenic plants. Nat. Biotechnol. 19, 157-161. 